\title{
Assessment of paranasal sinuses in Magnetic Resonance Imaging of brain
}

\author{
Surendra Maharjan
}

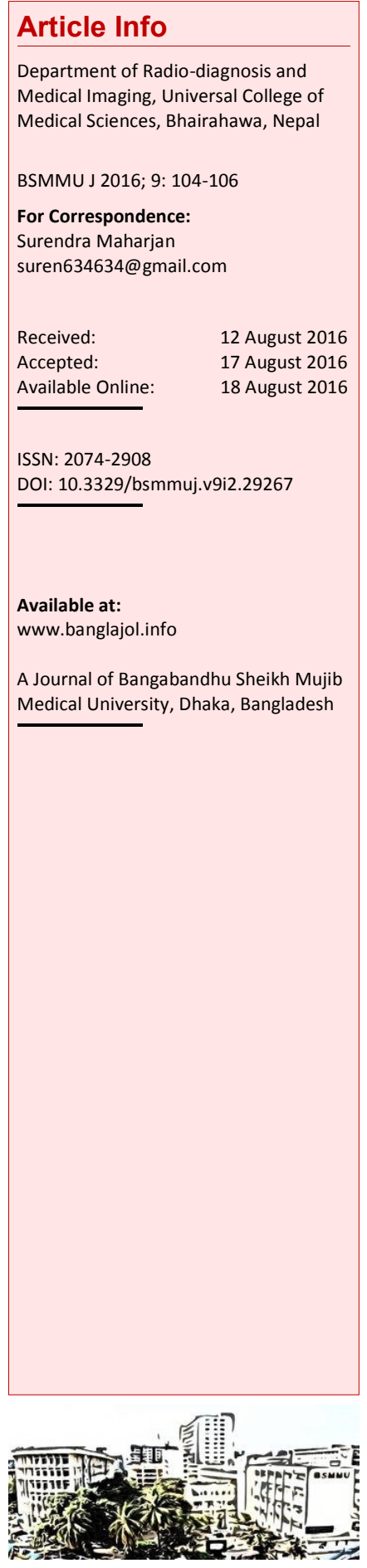

\begin{abstract}
Headache is the most common indication for Magnetic Resonance Imaging (MRI). But the minor ailments like paranasal sinusitis is often overlooked as it does not have specific discriminative characteristic and can imitate each type of primary headache. The purpose of this study was to evaluate the paranasal sinuses in patients undergoing MRI of brain. A cross-sectional hospitalbased study of 118 patients was done from April to September 2014. The images were evaluated for presence of any abnormal findings in sinuses. A random probabilistic sampling was carried out. The sinuses were normal in 51 patients and abnormal in 67 patients. The most common abnormality was mucosal thickening followed by polyp, cyst, mass and fluid level respectively. 50 of $67(74.6 \%)$ positive findings observed as mucosal thickening while polyp was seen in 13 patients $(28.4 \%)$. Similarly, both mass and fluid level was seen in $3(4.5 \%)$. The chi-square test showed no association between headache and abnormal sinus findings.
\end{abstract}

\section{Introduction}

Magnetic Resonance Imaging (MRI) is a highly sensitive medical imaging modality in the evaluation of various symptomatic conditions of the brain. MRI of the brain is a promising imaging test due to its best inherent contrast differences between various tissue structures. Mainly, inflammatory rhinosinusitis and suspected mass lesion are the two imaging conditions in paranasal sinuses. 1 In clinical practice, MRI of sinuses is performed in mass lesions, infections with cerebral and orbital complications. So, paranasal sinuses image is acquired under limited scans to determine the incidence and prevalence of sinus diseases. Computed Tomography (CT) scanning is the imaging method of choice for the paranasal sinuses.2 However, MRI is currently used for evaluation of sinus disease in limited study in combination with brain scanning.

The objective of this study are to determine the sinus pathology in patients undergoing MRI of brain, to classify the prevalence of sinus findings according to age, gender and clinical symptoms, to correlate the sinus findings with clinical symptoms, i.e. headache and to determine the type and location of sinus pathology.

\section{Materials and Methods}

This study was a prospective, observational, hospital based study conducted from April to
September 2014. A total of 118 patients referred by physician for MRI examination of brain were included in the study. MRI scans of the patients were performed in 0.3 Tesla Airis Vento Hitachi machine according to the standard protocols of the department. The scans were taken in T2 Axial, T1 Axial, Fluid Attenuated Inversion Recovery (FLAIR) Axial, T2 Coronal and T1 Sagittal sequences. The limited images of paranasal sinuses in these sequences were evaluated for the study.

T2 weighted axial images and FLAIR axial images of brain was obtained by positioning slices parallel to the bicommisural line, the line joining the anterior and posterior commissure. T1 axial sequence was also planned in similar way. The sagittal T1WI and the coronal T2WI were obtained. In sagittal scout image, the center of FOV was fixed at mass a intermedia whereas in coronal localizer, the scan lines were made parallel to the interhemispheric fissure. In axial T1 and T2 images, the normal maxillary, frontal, ethmoid and the sphenoid sinuses were clearly visualized as dark signal void. In midsagittal image, clear anatomic details of the paranasal sinuses were obtained. In coronal plane, the sinus pathology was best depicted.

\section{Results \\ Age distribution}

Age of the patients ranged from 15 to 89 years. 
The highest number of patients was in 15-30 years age group $(29.7 \%)$ followed by $30-45$ years age group $(28.8 \%)$. The lowest numbers of patients were in age group $75-90$ years of age comprising $4.2 \%$. In $45-60$ age group, there were 26 patients $(22.0 \%)$. Similarly, in 60-75 age group, there were only 18 patients $(15.3 \%)$. The mean age of the patients was $(43.2 \pm 18.2)$ years.

\section{Gender distribution}

Among the total of 118 patients, 57 were female and 61 were male.

\section{Distribution of sinus findings}

Among 118 scans, the paranasal sinuses were normal in $51(43.2 \%)$ patients. So, the number of patients with abnormal sinus finding was 67 . The abnormal sinus findings were categorized into four groups, namely mucosal thickening, polyp or retention cyst, mass and fluid level. 50 out of 67 (74.6\%) patients showed mucosal thickening; 19 out of $67(28.4 \%)$ showed polyp or retention cyst; 3 patients $(4.5 \%)$ demonstrated mass and 3 patients $(4.5 \%)$ had fluid level in the sinuses. Mucosal thickening was the most common incidental finding, and both mass and fluid level were least frequent.

Some patients had more than one finding. The second most common finding was cyst.

Among the 67 patients with abnormalities in paranasal sinuses, 50 had mucosal thickening. Maxillary sinus was the most common sinus involved in mucosal thickening, found in 39 of the 50 (78.0\%). Frontal sinus was the least involved sinus found in only 2 of the $50(4.0 \%)$. Some of the patients had sinus involvement at multiple sites. Among the 39 patients with maxillary sinus involvement, 21 $(53.8 \%)$ had bilateral sinus mucosal thickening, 12 $(30.8 \%)$ patients had left maxillary sinus thickening and $6(15.4 \%)$ had right maxillary thickening. There were 4 patients with mucosal thickening of ethmoid air cells, of which 4, 3 had in posterior and 1 had in anterior ethmoid air cells. 6 patients had sphenoid sinus mucosal thickening and 2 had the pathology in frontal sinus.

The maxillary is the most commonly affected sinus among all four sinuses. The ethmoid, sphenoid and frontal sinuses are less commonly affected sinuses.

Mass was seen only in ethmoid sinus in 3 patients. The largest size of mass was $44.6 \mathrm{~mm} \times 36.4 \mathrm{~mm}$. The second most common abnormality was polyp which was seen in 19, out of which 13 were present in maxillary sinus and 1 was in ethmoid sinus.

The mucosal thickening, which was measured in millimeters $(\mathrm{mm})$ are grouped into four categories. Maximum patients (48) had the thickness of $0-5$ $\mathrm{mm}, 19$ patients had mucosa of $5-10 \mathrm{~mm}, 3$ had 10-
$15 \mathrm{~mm}$ and only one patient had the maximum thickness of $15-20 \mathrm{~mm}$.

Retention cyst in the sinuses was seen in 19 patients. It was seen in left maxillary sinuses of 15 patients and right maxillary of 4 patients.

The size of cyst varied from $5.8 \mathrm{~mm} \times 4.8 \mathrm{~mm}$ to 34.4 $\mathrm{mm} \times 26.2 \mathrm{~mm}$ on left side and $15 \mathrm{~mm} \times 10 \mathrm{~mm}$ to $35 \mathrm{~mm} \times 20 \mathrm{~mm}$ on right side. The largest of the polyp was seen in left maxillary sinus.

Deviated nasal septum was deviated towards left side in 7 patients, and towards right side in 2 patients. Concha bullosa was not seen in our study, neither was there any asymmetry of the nasal turbinates.

\section{Distribution according to clinical information}

Among the 118 patients, the most common clinical indication for MRI brain was headache which was present in 67 patients $(56.8 \%)$, followed by seizure in 15 patients $(12.7 \%)$, suspected mass in 9 patients $(7.6 \%)$, follow up scan in $2(1.7 \%)$. The Other indications included neuro-degenerative diseases, meningitis, encephalitis etc. in 25 patients (21.2\%).

\section{Association between headache and abnormal sinus} findings

The Chi-square test was performed to observe the association between the headache and abnormal sinus pathology detected in the MRI scans. The test showed no association at 5\% level of significance. With p-value of 0.305 , we concluded that sinus pathology was not the cause of headache in these patients. The sinus pathology, thus, was incidental finding.

\section{Discussion}

A hospital based cross-sectional study was done in paranasal sinuses of MRI images of 118 patients. These patients were referred for MRI scan of brain for various clinical indications. Among 118 scans, 51 showed normal paranasal sinuses. However, 67 showed various findings.

Age of the patients ranged from 15 to 89 years. The mean age of the patients was $(43.2 \pm 18.2)$ years. The age distribution was similar to the study done by Huang and his colleagues. 3 Among the total of 118 patients, 57 were female and 61 were male. This was nearly similar to study done by Katzman et al. $\underline{4}$

Among 118 scans, the paranasal sinuses were normal in 51 patients. So, the number of patients with abnormal sinus finding was 67 . Katzman et al. found sinus disease in $13.2 \%$ of the 1000 participants. 4 The paranasal sinuses findings are significantly higher as compared to this literature. Huang and his colleagues detected incidental fin- 
dings in $47.0 \%$ (71/151). 3 They also retrospectively reviewed 129 patients in $\mathrm{fMRI}$ and found incidental abnormalities in $48(37.2 \%)$ patients. 5 Again, the incidental finding in our study is relatively more.

The Mind Research Network (MRN), an independent non-profit research institute, had reviewed approximately $6,000 \mathrm{MR}$ brain scans, and they reported $2722(36 \%)$ incidental finding. 6 This result also didn't match our results. The abnormal findings are much higher in our study. However, the patients didn't require immediate and urgent treatment.

In the present study, the abnormal sinus findings were categorized into four groups, namely mucosal thickening, polyp or retention cyst, mass and fluid level. 50 out of $67(74.6 \%)$ patients showed mucosal thickening; 19 out of $67(28.4 \%)$ showed polyp or retention cyst; 3 patients $(4.5 \%)$ demonstrated mass and 3 patients $(4.5 \%)$ had fluid level in the sinuses.

Mucosal thickening was the most common incidental finding, and both mass and fluid level were least frequent. Some patients had more than one finding.

Maxillary sinus was the most common sinus involved in mucosal thickening. The ethmoid, sphenoid and frontal sinuses are less commonly affected sinuses.

The second most common finding was retention cyst/polyp. The polyp in the sinuses was seen in 19 patients. It was seen in left maxillary sinuses of 15 patients and right maxillary of 4 patients. The largest of the polyp was seen in left maxillary sinus.

Mass was seen only in ethmoid sinus in 3 patients. The largest size of mass was $44.6 \mathrm{~mm} \times 36.4 \mathrm{~mm}$. The second most common abnormality was polyp which was seen in 19, out of which 13 were present in maxillary sinus and 1 was in ethmoid sinus.

Some of the patients had sinus involvement at multiple sites.

McNeil et alz also found no statistical association in between clinical symptoms of paranasal sinuses and MRI paranasal findings. The present study showed no association between headache and abnormal sinus pathology at $5 \%$ level of significance with pvalue of 0.305 . We concluded that sinus pathology did not cause headache in these patients. The sinus pathology thus was incidental finding.

\section{Conclusion}

In the present study of 118 patients referred for MRI Brain, incidental findings in paranasal sinuses were demonstrated in 67 cases $(56.6 \%) ; 50$ cases $(74.6 \%)$ of these incidental findings were mucosal thickening and required no further actions. However, 3 cases $(4.8 \%)$ demonstrated mass and thus require appropriate referral to other specialist. There was no association between the headache and the abnormal pathology demonstrated in sinuses.

\section{References}

1. Del JM, Gaudio MF. Sinus headache vs migraine. Emory University School of Medicine, Department of Otolaryngology, 2013 (Presentation slides).

2. Rick Balys MF. Sinus headache: Revisiting the diagnosis. Halifax, Nova Scotia, Dal Housie University, 2012.

3. Alles J, Rosen AC, Huang, et al. Ethical consideration of incidental findings on adult brain MRI in research. Neurology 2004; 62: 888-90.

4. Katzman GL, Dagher AP, Patronas NJ. Incidental findings on brain magnetic resonance imaging from 1000 asymptomatic volunteers. JAMA. 1999; 282: 36 -39 .

5. Illes J, Desmond JE, Huang LF, Raffin TA, Atlas SW. Ethical and practical considerations in managing incidental findings in functional magnetic resonance imaging. Brain Cogn. 2002: 50: 358-65.

6. Shoemaker JM. Expect the unexpected. New Mexico, Mind Research Network (MRN), 2010.

7. McNeill E, O'Hara J, Carrie S. The significance of MRI findings for non-rhinological disease. Clin Otolaryngol. 2006; 31: 292-96. 